Gefangenschaft entwichenes Stück, was gerade bei diesen so häufig gekäfigten Vögeln nicht ausgeschlossen wäre, handelt. Sichere Nachweise von etwaigen Brutvorkommen sind bis bisher also nicht erbracht.

$\mathrm{S} \mathrm{cha} \mathrm{low} \mathrm{ist} \mathrm{das} \mathrm{zweitgenannte} \mathrm{im} \mathrm{Berl.} \mathrm{Mus.} \mathrm{befindliche}$ Belegexemplar unbekannt geblieben, da er es in seinen Arbeiten nirgends erwähnt. - Hartert (l. c. p. 737) bringt bei der geographischen Verbreitung keinerlei Mitteilungen von Vorkommen aus der Mark Brandenburg.

\title{
Markierung von Rauhfufsbussarden (Archibuteo lagopus L.) in Schwedisch-Lappland Sommer 1912 und Markierung von schwedischen Flufs-Seeschwalben (Sterna himundo L.).
}

Von Prof. Dr. L. A. Jägerskiöld,

Intendant der Zoolog. Abteilung des Museums zu Gothenburg.

Die von dem Biologischen Verein zu Gothenburg 1911 angefangenen Markierungen von schwedischen Zugvögeln wurden auch 1912 fortgesetzt und zwar wurden auch dies Jahr Rauhfursbussarde beringt. Herr Konservator G. Kihlén hat die recht schwierige Arbeit ausgeführt. Es wurden im Juli 78 junge Vögel in den Nestern mit Ringen (mit Nummern zwischen 200-299 nebst Aufschrift Museum Göteborg Sweden) versehen. Sämtliche Nester befanden sich in der Gegend etwa 25 Kilometer südlich von Kiruna [K a der Karte] (etwa $20^{\circ} 13^{\prime} 29^{\prime \prime}$ O. v. Greenwich und $67^{\circ} 38^{\prime} \mathrm{N}$. Br.).

Im Sommer 1912 waren die Lemminge sehr selten und auch von den anderen kleinen Nagern war kein grofser Bestand da. Daher sind wahrscheinlich in jedem Nest 1-3 Junge durch Hunger nach der Beringung zu Grunde gegangen. Die Wahrscheinlichkeit hiervon geht dadurch hervor, dafs man beim Beobachten einzelner Nester einige Zeit nach der Markierung daselbst verhungerte Junge vorgefunden. Daraus folgt aber, dafs man leider von Anfang an keinen so guten Erfolg erwarten könnte wie aus den Markierungen im Jahre 1911. - Es sind jedoch 7 der beringten Vögel angemeldet woŕden. Hierzu kommt noch einer der 1911 Markierten.

Das nachstehende Verzeichnis nebst der beigegebenen kleinen Karte gewährt eine Übersicht der Befunde. Auf die Karte sind nur die während des Winters 1912-1913 angemeldeten Vögel und ihre Fundorte mit Datum und einem $\bigcirc$ ausgezeichnet eingetragen, wegen der die 1911 beringten und im Winter 19111912 angemeldeten Vögel vergl. die Karte auf S. 381 Jahrg. 1913. 
Markierung von Rauhfufsbussarden und Flufs-Seeschwalben. 269

Ring-Nr. 254. Fundstelle: Killinge-Fjällåsen, am 4. IX. 1912.

Finder: Hugo Svärd.

Nr. 227. Fundstelle: Bollsta bruk Ångermanland, am 31. VIII.

1912. Finder: Abr. Selin.

Nr. 274. Fundstelle: Frantsila, Kirchspiel Siikajoki Uleáborgs län, Finland, am 14. IX. 1912. Finder: Matti Lehto.

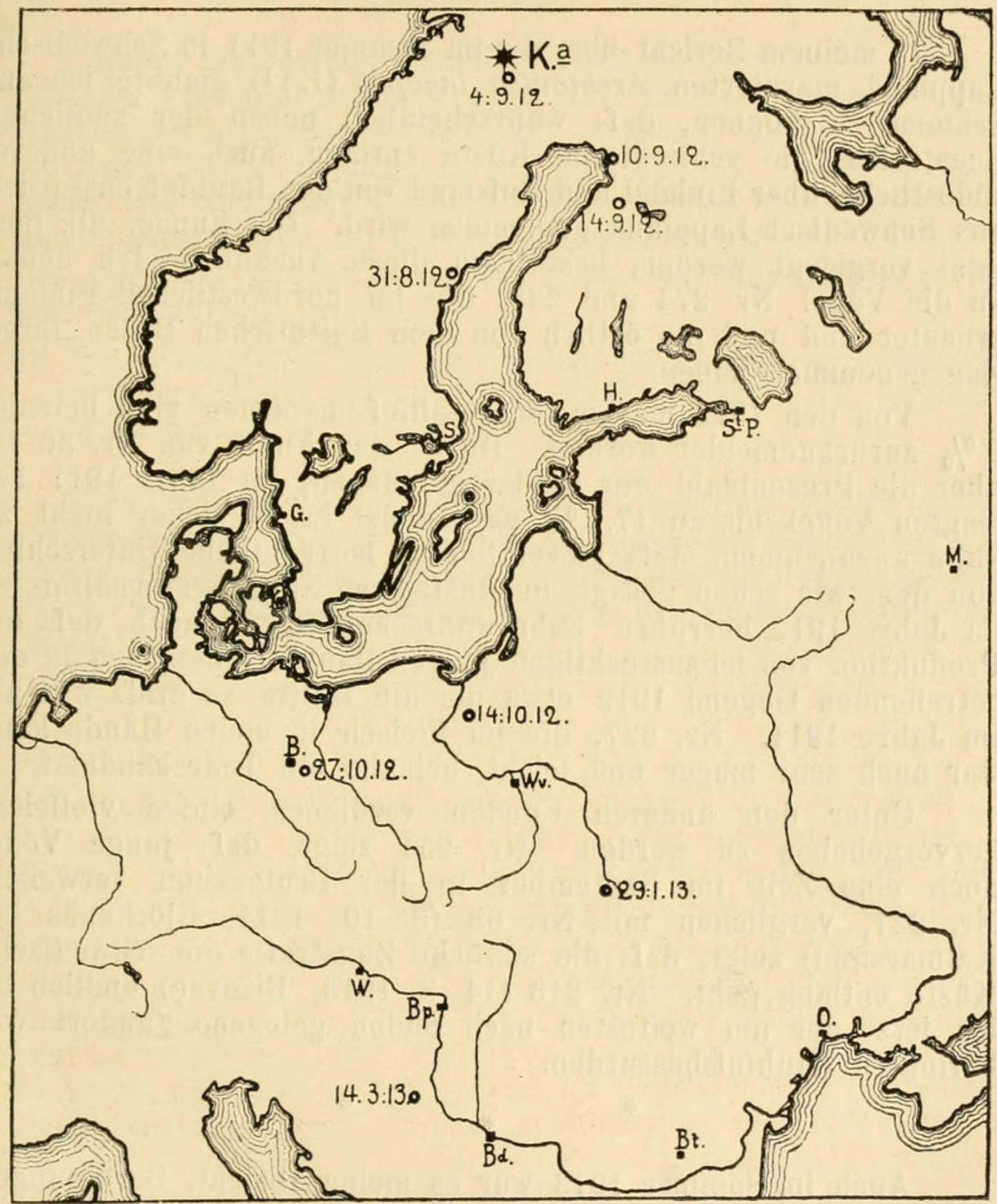

Nr. 252. Fundstelle: Storkow in der Mark, am 27. X. 1912. Finder: Rechtsanwalt Alfred Koeppen.

Nr. 228. Fundstelle: Jokikylã, Kirchspiel Hankipudas Uleåborgs län, Finland, am 10. IX. 1912. Finder: Hermanni Juvani. (Mitgeteilt durch J. A. Palmén.) 
Nr. 279. Fundstelle: Gutsherrschaft Odnów (15 km von Lemberg), Galizien, am 29. I. 1913. Finder: Emil Ritter v. Obertynski.

Nr. 213. Fundstelle: Bizovaç, Slavonien, Ungarn, am 14. III. 1913. Finder: Forstamtsadjunkt J. Fichtner.

Nr. 36. Fundstelle: Herzogswald, Schönberg, Kreis Rosenberg, Westpreufsen, am 14. X. 1912. Finder: Oberförster Grube.

In meinem Bericht über die im Sommer 1911 in SchwedischLappland markierten Archibuteo lagopus (L.) ${ }^{1}$ ) glaubte ich annehmen zu können, dafs wahrscheinlich neben der südlichen Zugstrafse, die schwedische Küste entlang, auch eine andere, südöstliche, über Finland und Rufsland von den Rauhfufsbussarden aus Schwedisch-Lappland genommien wird. Die Funde, die hier jetzt vorgelegt werden, bestätigen diese Annahme. Ich denke an die Vögel Nr. 274 und 228, die im nordwestlichen Finland erbeutet sind und die östlich von dem Bottnischen Busen ihren Zug genommen haben.

Von den 1912 markierten Rauhfufsbussarden sind beinahe $9 \%$ zurückgemeldet worden. Durch den Fund von Nr. 36 ist aber die Prozentzahl der wiedergefundenen, im Jahre 1911 beringten Vögel bis $\mathrm{zu} 17,1 \%$ gestiegen. Es ist sicher nicht zu kühn anzunehmen, dafs dieser höchst beträchtliche Unterschied von den, wie schon gesagt, ungünstigeren Nahrungsverhältnissen im Jahre 1912 herrührt. Sehr wahrscheinlich ist auch, dafs die Produktion von lebenstüchtigen jungen Rauhfufsbussarden in der betreffenden Gegend 1912 etwa um die Hälfte so grofs war als im Jahre 1911. Nr. 227, die im Fleisch in meine Hände kam, war auch sehr mager und leicht, beinahe ein Todeskandidat.

Unter den anderen Funden verdienen einige vielleicht hervorgehoben zu werden. Nr. 254 zeigt, dafs junge Vögel noch eine Zeit im September in der Brutgegend verweilen. Nr. 227, verglichen mit Nr. 68 (8. 10. 1911, Björkenäs bei Kalmarsund) zeigt, dafs die südliche Zugstrafse die schwedische Küste entlang geht. Nr. 213 (14. 3. 1913, Bizovaç) endlich ist bis jetzt der am weitesten nach Süden gelegene Fundort von beringten Rauhfufsbussarden.

Auch im Sommer 1913 war es meine Absicht, Beringungen von Rauhfufsbussarden durch die Hilfe des Herrn Konservators G. Kihlén ausführen zu lassen. Unser Vertreter in der Gegend von Kiruna, Herr Hugo Swärd, teilte mir aber mit, dafs die Rauhfufsbussarde zwar wie gewöhnlich im Frühling (Anfang.

1) Diese Zeitschrift, Aprilheft 1913. 
Mai) ankamen, nach kurzer Frist aber wieder davon gezogen sind. Daher waren sehr wenige oder, vielleicht richtiger, so gut wie keine Nester zu finden. Dies hing natürlich davon $a b$, dafs von Lemmingen und anderen kleinen Nagern fast nichts zu sehen war. Die Vögel zogen daher nach besseren Jagdgebieten, um sich dort fortzupflanzen.

Damit der Sommer doch nicht ohne irgend welche Beringungsversuche verlaufen sollte, wurden statt Rauhfufsbussarden FlufsSeeschwalben (Sterna hirundo L) markiert, und zwar wurden 190 junge Vögel zwischen 3. VII. und 16. VII. in den Scheeren unweit von Gothenburg beringt. Auch diesmal verdanke ich dem Herrn Konservator G. Kihlén die Ausführung der Feldarbeit. Von den Flufs-Seeschwalben ist bis jetzt nur eine einzige zurückgemeldet worden, nämlich:

Nr. 21. Fundstelle: Baie de Somme, im nördlichen Frankreich, an dem Kanal, am 27. VIII. 1913. Finder: G. le Duchat d'Aubigny.

Ich hoffe aber, dafs mit der Zeit mehrere werden zurückgemeldet werden. Dem sei, wie ihm wolle, die Beringungen von schwedischen Zugvögeln werden hoffentlich durch den Biologischen Verein zu Gothenburg auch in den kommenden Jahren fortgesetzt werden. 


\section{$2 \mathrm{BHL}$ Biodiversity Heritage Library}

1914. "Markierung von Rauhfufsbussarden (Archibuteo lagopus L.) in Schwedisch-Lappland Sommer 1912 und Markierung von schwedischen Flufs-Seeschwalben (Sterna hirundo L.)." Journal $f u$

r Ornithologie 62, 268-271. https://doi.org/10.1007/bf02096341.

View This Item Online: https://www.biodiversitylibrary.org/item/43524

DOI: https://doi.org/10.1007/bf02096341

Permalink: https://www.biodiversitylibrary.org/partpdf/142795

\section{Holding Institution}

MBLWHOI Library

\section{Sponsored by}

MBLWHOI Library

\section{Copyright \& Reuse}

Copyright Status: No known copyright restrictions as determined by scanning institution.

This document was created from content at the Biodiversity Heritage Library, the world's largest open access digital library for biodiversity literature and archives. Visit BHL at https://www.biodiversitylibrary.org. 